\title{
Unilateral Pedicular Screws and Interbody Lumbar Spine Fusion in Unilateral Degenerative Pathology (Technique and Outcome)
}

\author{
Shafik Tahseen El Molla*, Hisham Anwer, Hatem Sabry \\ Neurosurgery Department, Ain Shams University, Cairo, Egypt \\ Email address: \\ drshafikelmolla@gmail.com (S. T. El Molla), hishambando@yahoo.com (H. Anwer), sabrymd@gmail.com (H. Sabry) \\ ${ }^{*}$ Corresponding author \\ To cite this article: \\ Shafik Tahseen El Molla, Hisham Anwer, Hatem Sabry. Unilateral Pedicular Screws and Interbody Lumbar Spine Fusion in Unilateral \\ Degenerative Pathology (Technique and Outcome). International Journal of Neurosurgery. Vol. 4, No. 2, 2020, pp. 34-40. \\ doi: $10.11648 /$ j.ijn.20200402.13
}

Received: June 22, 2020; Accepted: July 25, 2020; Published: August 10, 2020

\begin{abstract}
Introduction: Lumbar degenerative diseases (LDD) is considered a common disease. Lumbar pedicular screw fixation and interbody fusion is one of the commonly used and effective surgical method in management of single-level lumbar degenerative diseases. Although bilateral pedicular screw (PS) fixation after lumbar interbody fusion is accepted as a standard surgical procedure providing rigid fixation with a great biomechanical stability and clinical benefits, the rigidity of bilateral (PS) fixation can cause device-related osteoporosis of the vertebrae which makes the adjacent segment prone to load and motion-induced degeneration. Therefore, the concept of using less rigid systems of fixation has been advocated. Aim of study: To evaluate effectiveness of unilateral pedicular screws fixation and interbody fusion in unilateral degenerative spine pathology. Patients and methods: This study included 34 patients with single level degenerative lumbar spine disease who were subjected to transforaminal interbody lumbar fusion (TILF) and unilateral transpedicular screws fixation between July 2017 and March 2019 in Ain Shams University hospitals. Results: The study was conducted on 34 patients with age ranging between 32-56 years (mean $45.12 \pm 7.80$ years). There were 17 male and 17 female in this study. When comparing VAS back pain preoperatively and at postoperative intervals of 1,6 and 12 months, it showed highly significant improvement (P-value 0.000 ). Also when comparing VAS of leg pain preoperatively and at postoperative intervals of 1,6 and 12 months duration, it showed highly significant improvement (P-value 0.000). ODI preoperatively and at postoperative intervals of 1,6 and 12 months showed also highly significant improvement (P-value 0.000 ). Regarding the fusion rate, at 6 months postoperative $61.8 \%(21)$ of the patients had fusion grade $1,26.5 \%$ (9) of the patients had fusion grade 2 and $11.8 \%$ (4) of the patients had fusion grade 3 . While at 12 months postoperative the percent of grade 1 fusion increased to become $85.3 \%$ ( 29 patients) and the rest were grade 2 fusions (14.7\%) with no patients with grade 3 fusion. Conclusion: Unilateral pedicular screws and interbody fusion is a good modality of treatment for unilateral degenerative spine pathology.
\end{abstract}

Keywords: Lumbar Fixation, Unilateral, Interbody Cage Fusion, Degenerative Lumbar Spine

\section{Introduction}

Lumbar degenerative diseases (LDD) is considered a common disease which includes prolapsed lumbar disc, degenerative instability, spondylolisthesis of lumbar vertebrae, lumbar spinal stenosis, and degenerative scoliosis. [1]

Lumbar pedicular screw fixation and interbody fusion is one of the commonly used and effective surgical method in management of single-level lumbar degenerative diseases
(LDD), such as discogenic pain, spondylolisthesis and lumbar spinal canal stenosis associated with spinal deformities. Bilateral pedicle screw fixation and interbody fusion with cage (BPSFC) is widely used for achieving lumbar spinal fusion. It can cause initial stability, correct deformities, maintain the intervertebral disc height, improve interbody fusion, and fasten the recovery process from spinal surgery [2].

Although bilateral pedicular screw (PS) fixation after lumbar interbody fusion is accepted as a standard surgical 
procedure providing rigid fixation with a great biomechanical stability and clinical benefits, the rigidity of bilateral (PS) fixation can cause device-related osteoporosis of the vertebrae which makes the adjacent segment prone to load and motion-induced degeneration. Therefore, the concept of using less rigid systems of fixation has been advocated. Some recent clinical and biomechanical studies on the effectiveness of unilateral (PS) fixation have showed that a reliable fusion with fewer pedicular screws can be achieved. [3]. Aim of this study is to evaluate effectiveness of unilateral pedicular screws fixation and interbody fusion in unilateral degenerative spine pathology.

\section{Patients and Methods}

This study included 34 patients with single level degenerative lumbar spine disease who were subjected to transforaminal interbody lumbar fusion (TILF) and unilateral transpedicular screws fixation between July 2017 and March 2019 in Ain Shams University hospitals.

\subsection{Inclusion Criteria}

1. Patients with low back pain and unilateral radiculopathy caused by single level degenerative lumbar spine disease as lumbar disc herniation whether recurrent or denovo and grade 1 spondylolisthesis.

2. No response to medical treatment for 3 months.

3. Failure to perform daily activities.

\subsection{Exclusion Criteria}

1. Presence of bilateral radiculopathy.

2. Affection of more than single level.

3. Spondylolithesis greater than grade 1 .

4. Presence of other pathology as trauma, tumor or infection.

All patients were subjected to full history taking and thorough neurological examination. Visual analogue scale (VAS) for leg and back pain is recorded pre-operatively and at 1,6,12 months interval post-operatively. The Oswestry Disability Index (ODI) is calculated pre-operatively and at $1,6,12$ months interval.

All patients have undergone pre-operative lumbosacral $\mathrm{x}$ ray lateral, flexion and extension views to detect any instability. Pre-operative MRI is done to determine the affected level. Pre-operative disc height is calculated by this formula: Disc height $=($ Anterior height + Posterior height $) / 2$.

\subsection{Surgical Technique}

The patient is placed in the prone position. A Foley catheter is inserted preoperatively to ensure that bladder distension does not cause any increase in the intra-abdominal pressure during the surgery. Following administration of perioperative antibiotics and skin and paraspinal muscles infiltration with $1 \%$ bupivacaine, a midline skin incision of approximately $10 \mathrm{~cm}$ is made according to the pathologic disc space. The lumbosacral fascia is opened along the midline. The spinous processes and the laminae above and below the level of the pathology were exposed. The paraspinal muscles would be retracted laterally to minimize damage, and to expose the posterior joint. Pedicle screws are then placed on the symptomatic side under fluoroscopic guidance. A complete unilateral facetectomy was performed on the symptomatic side which permitted complete neural decompression and a direct approach to the pathological disc space with minimal neural retraction. The nerve root exiting below the superior pedicle might be at the greatest risk for injury, especially with the placement of intervertebral instrumentation. Injury to the dorsal root ganglion might result in permanent neuropathic pain that could be resistant to all treatment modalities.

The epidural venous plexus over the dorsal annulus has to be meticulously cauterized to maintain a dry field to reduce blood loss. In the procedure the spinous process, supraspinatus and interspinous ligaments and the contralateral facet joints, remained untouched. Exposure of the working window is then done which consists of the following: The traversing nerve root and thecal sac forming the medial border of the triangle, the exiting nerve root forming the proximal vertebral level forms the lateral border, the superior aspect of the pedicle of the distal vertebra forming the base of the triangle.

The disc space is entered, and a $7 \mathrm{~mm}$ intradiscal shaver is inserted parallel to the endplates, and rotated a number of times to ensure adequate preparation of the host graft site. Successful removal of the cartilaginous end-plates is an important step to ensure adequate fusion.

Once the disc space expansion has been achieved using interbody distraction, the pedicle screws could be tightened over a temporary rod to maintain the distracted position. The interbody space was then reconstructed by selecting an appropriate sized interbody cage. Proper sizing of the interbody cage depends on disc height at the level above and below the affected disc. The interbody cage was then packed with autogenous cancellous bone or any appropriate graft substitute. Cage position was confirmed using image intraoperatively. Any distraction that has been temporarily applied has to be released after placement of the interbody cage. Rods were attached to the pedicle screws, and compression was applied to the construct. Before wound closure, a probe has to be used to confirm adequate space around the neural structures and to ensure that graft material has not migrated into the foraminal region.

Post-operatively lumbosacral x-ray is obtained at 1, 6 and 12 months interval and operated lumbar disc height is calculated with evaluation of fusion grade.

(Grade 1) Definite fusion: Definitive bony trabecular which bridges across the graft/host interface, there is no detectable motion on flexion-extension radiographs, with no gap at the interface.

(Grade 2) Probable fusion: No definitive bony trabecular is crossing, but with no detectable motion and no identifiable gap through the interface.

(Grade 3) Possible pseudarthrosis: No bony trabecular is crossing, no motion, but there is an identifiable gap at the interface. 
(Grade 4) Definite pseudarthrosis: No traversing trabecular bone, there is definitive gap at the interface, and with motion on flexion-extension radiographs.

Grade 1 and 2 were considered as successful fusions, while 3 and 4 were considered as failed fusions.

Data were collected, revised, coded and entered to the Statistical Package for Social Science (IBM SPSS) version 23. The quantitative data were presented as mean, standard deviations and ranges when parametric. Also qualitative variables were presented as number and percentages. The confidence interval was set to $95 \%$ and the margin of error accepted was set to $5 \%$. So, the p-value was considered significant as the following:

$\mathrm{P}>0.05$ : Non significant (NS)

$\mathrm{P}<0.05$ : Significant $(\mathrm{S})$

$\mathrm{P}<0.01$ : Highly significant (HS)

\section{Results}

The study was conducted on 34 patients with age ranging between $32-56$ years (mean $45.12 \pm 7.80$ years). There were 17 males and 17 females in this study. The reason for unilateral fixation and fusion was recurrent lumbar disc in $76.5 \%$ of patients, foraminal stenosis in $5.9 \%$ of patients, spondylolisthesis in $8.8 \%$ of patients and denovo lumbar disc prolapse in $8.8 \%$ of patients. There were $29(85.3 \%)$ patients with L4-5 disc level affection, $3(8.8 \%)$ patients with L5-S1 level affection and 2 (5.9\%) patients with L3-4 level affection. Operation time ranged from 100-140 min (mean $118.91 \pm 12.75)$ and the blood loss intraoperative was ranging from 200-600 cc (mean 405.88 \pm 118.55 ). Hospital stay ranged from 3-5 days. (table 1)

When comparing VAS back pain preoperatively and at postoperative intervals of 1,6 and 12 months, it showed highly significant improvement (P-value 0.000). Also when comparing VAS of leg pain preoperatively and at postoperative intervals of 1,6 and 12 months duration, it showed highly significant improvement (P-value 0.000). ODI preoperatively and at postoperative intervals of 1,6 and 12 months showed also highly significant improvement (P-value 0.000).

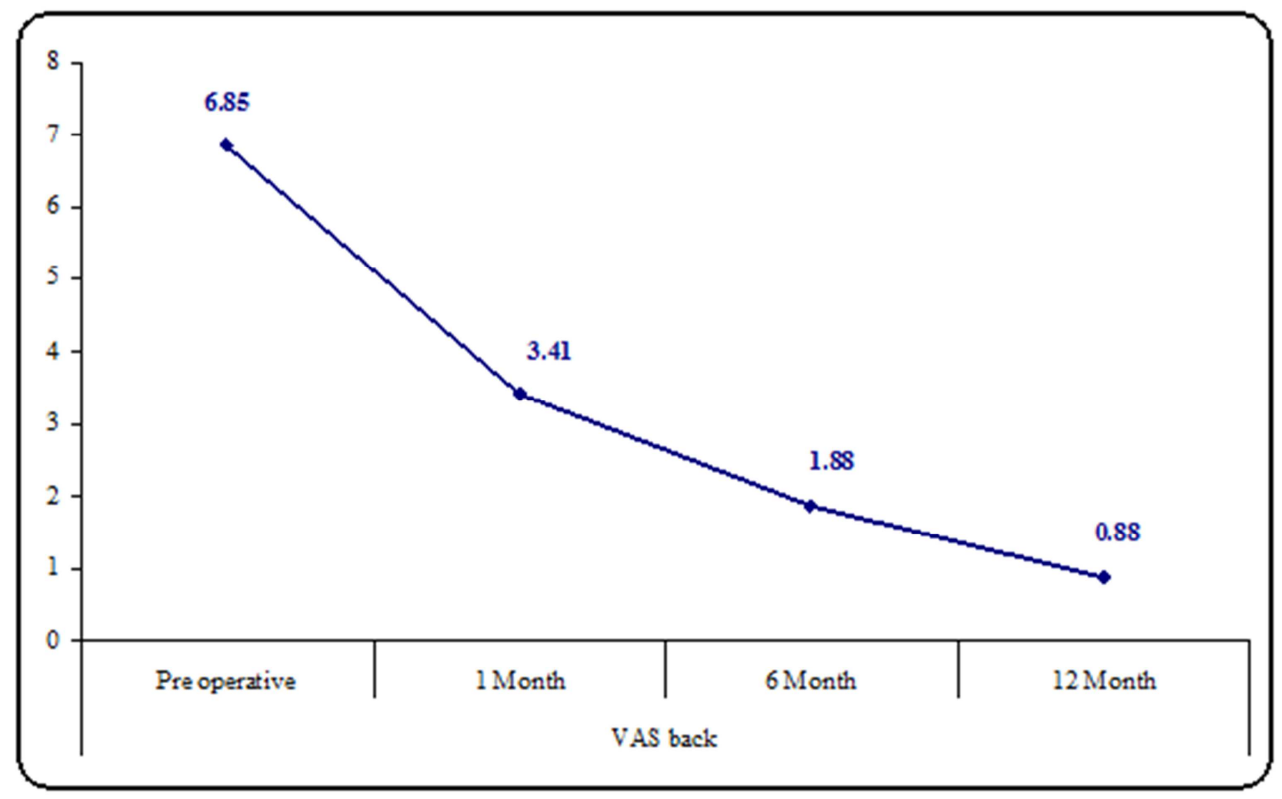

Figure 1. VAS of back pain preoperative and postoperative.

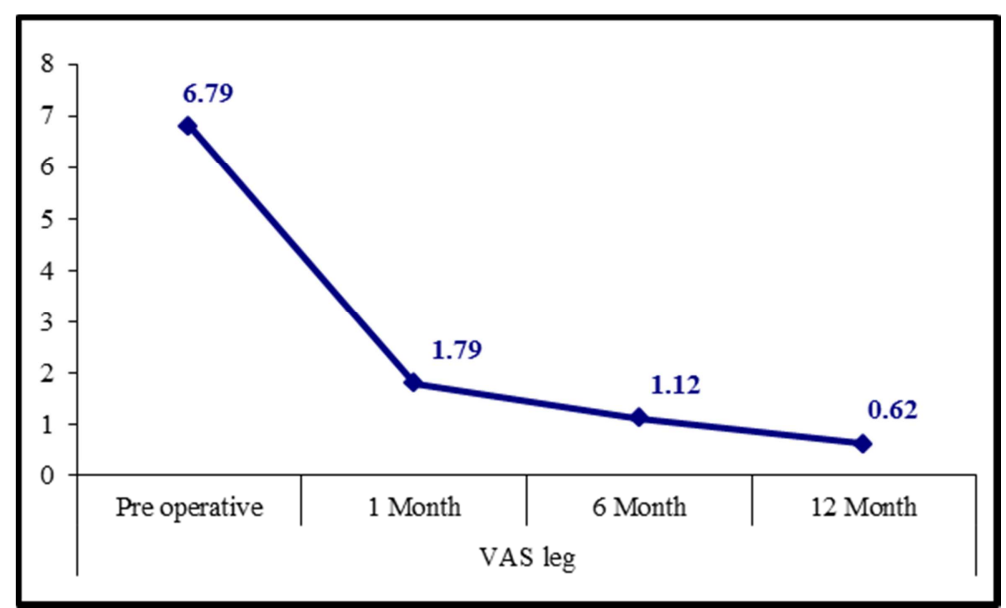

Figure 2. VAS of leg pain preoperative and postoperative. 


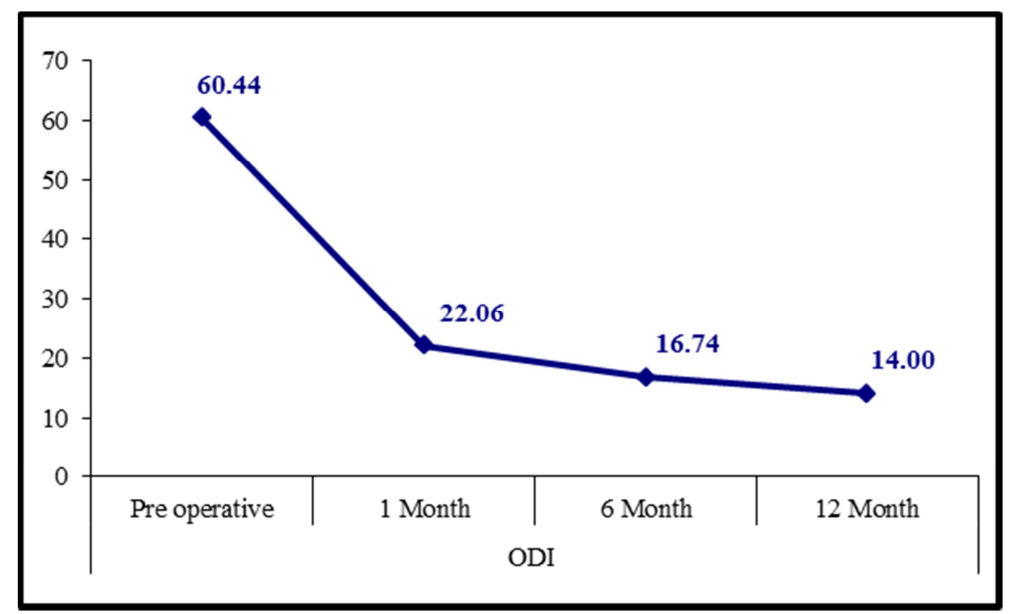

Figure 3. ODI preoperative and postoperative.

Table 1. Statistical analysis

\begin{tabular}{lll}
\hline & & No. $=\mathbf{3 4}$ \\
\hline \multirow{2}{*}{ Age years } & Mean \pm SD & $45.12 \pm 7.80$ \\
Sex & Range & $32-56$ \\
& Female & $17(50.0 \%)$ \\
& Male & $17(50.0 \%)$ \\
Diagnosis & Recurrent lumbar disc & $26(76.5 \%)$ \\
& Foraminal stenosis & $2(5.9 \%)$ \\
& Spondylolisthesis & $3(8.8 \%)$ \\
Level & Denovo lumbar disc & $3(8.8 \%)$ \\
& L4-L5 & $29(85.3 \%)$ \\
Operation time (min) & L5-S1 & $3(8.8 \%)$ \\
& L3-L4 & $2(5.9 \%)$ \\
Blood loss (ml) & Mean \pm SD & $118.91 \pm 12.75$ \\
& Range & $100-140$ \\
Duration of pain killers use (days) & Mean \pm SD & $405.88 \pm 118.55$ \\
& Range & $200-600$ \\
Hospital length of stay (days) & Mean \pm SD & $2.91 \pm 1.19$ \\
\hline
\end{tabular}

Segmental disc height means was $2.69 \pm 0.41 \mathrm{~mm}$ in 1 month postoperative, $2.10 \pm 0.38 \mathrm{~mm}$ in 6 months postoperative and $1.96 \pm 0.33 \mathrm{~mm}$ in 12 months postoperative.

Regarding the fusion rate, at 6 months postoperative $61.8 \%$ (21) of the patients had fusion grade 1, 26.5\% (9) of the patients had fusion grade 2 and $11.8 \%$ (4) of the patients had fusion grade 3 . While at 12 months postoperative the percent of grade 1 fusion increased to become $85.3 \%$ (29 patients) and the rest were grade 2 fusions $(14.7 \%)$ with no patients with grade 3 fusion (table 2).

Table 2. Fusion grade 6 and 12 months postoperatively.

\begin{tabular}{llll}
\hline Fusion grade & & No. & \% \\
\hline & 1 & 21 & $61.8 \%$ \\
6 month & 2 & 9 & $26.5 \%$ \\
& 3 & 4 & $11.8 \%$ \\
& 1 & 29 & $85.3 \%$ \\
12 month & 2 & 5 & $14.7 \%$ \\
& 3 & 0 & $0.0 \%$ \\
Chi-square test & & 6.423 & \\
P-value & & $0.040(\mathrm{~S})$ & \\
\hline
\end{tabular}

As for complications there were 2 patients (5.9\%) with incidental autotomy, 1 patient with cage migration (2.9\%) and 1 patient $(2.9 \%)$ with superficial wound infection. 

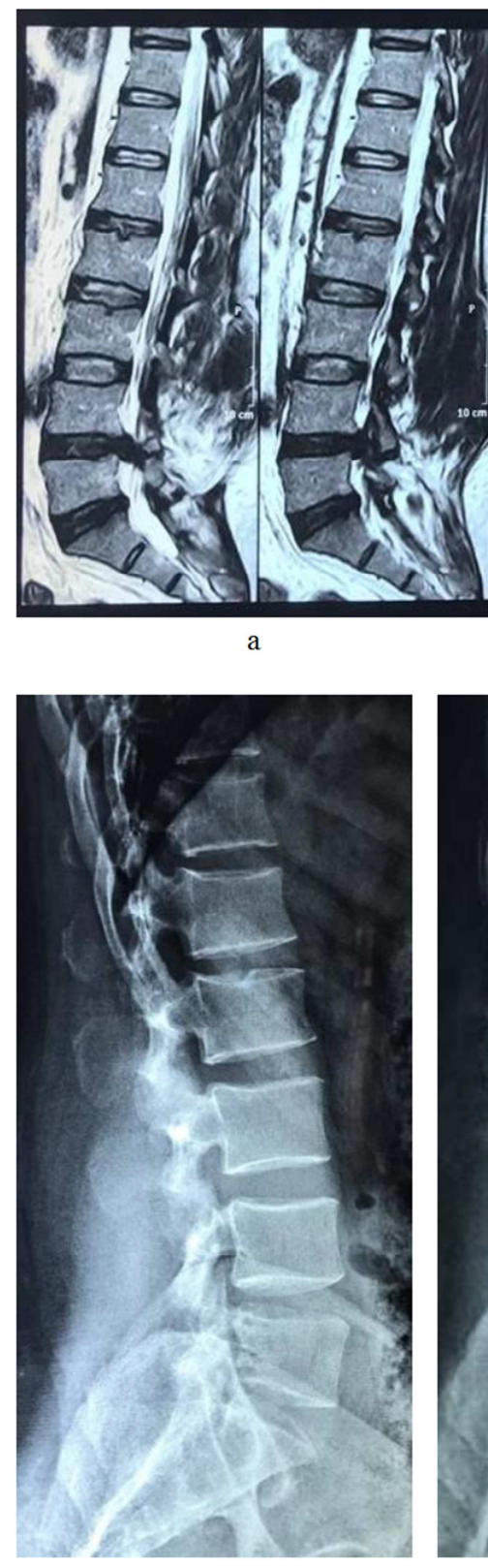

c

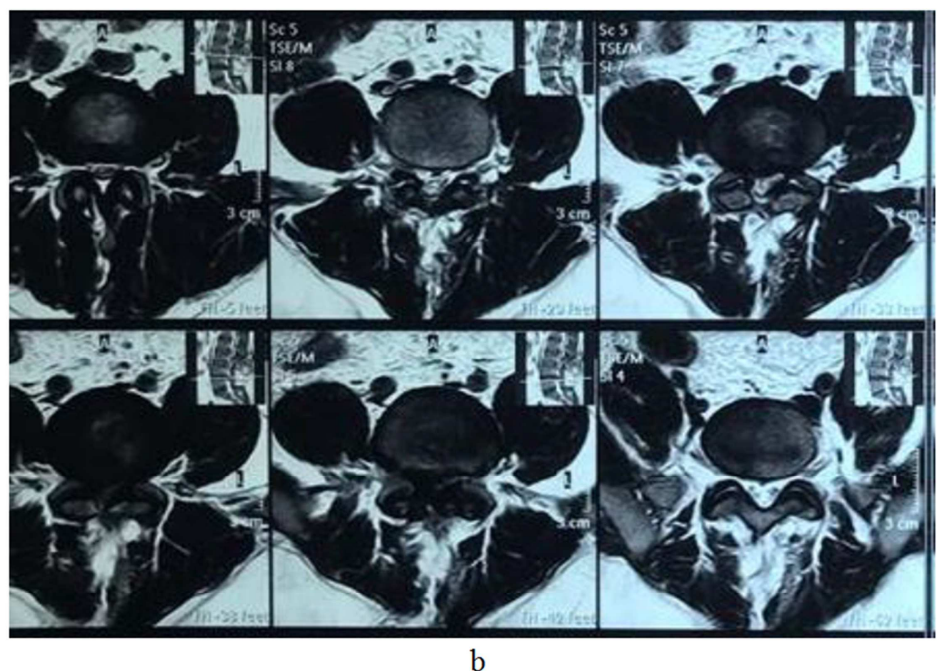

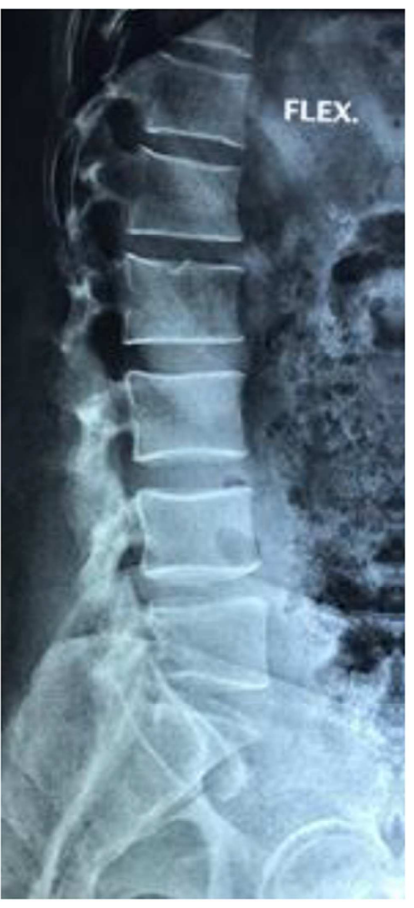

$\mathrm{d}$

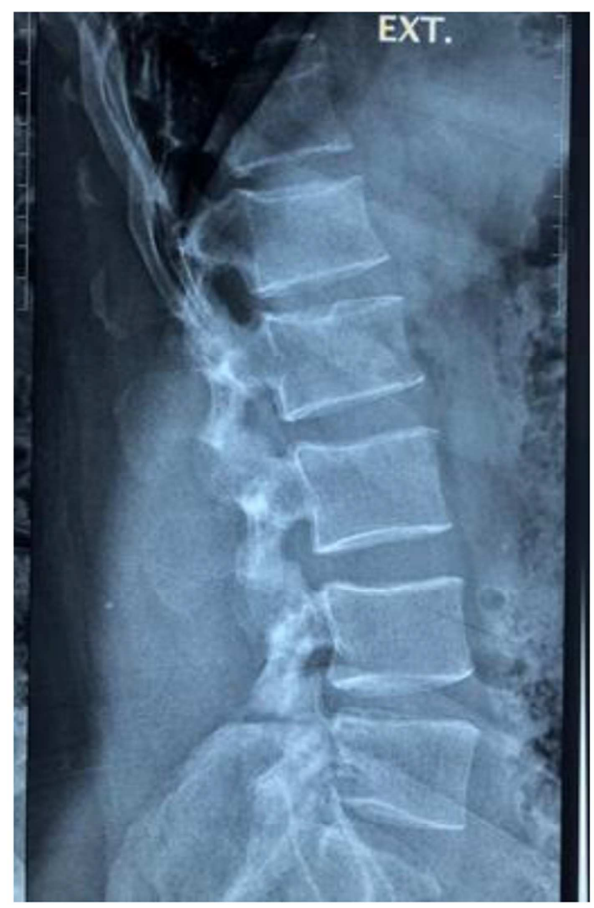

Figure 4. (a. $b, c, d$, e). Preoperative radiology of 46 years old, male patient, recurrent L4-5 disc prolapse, presented with LBP and Right sciatica of 6 months' duration without improvement by conservative methods.

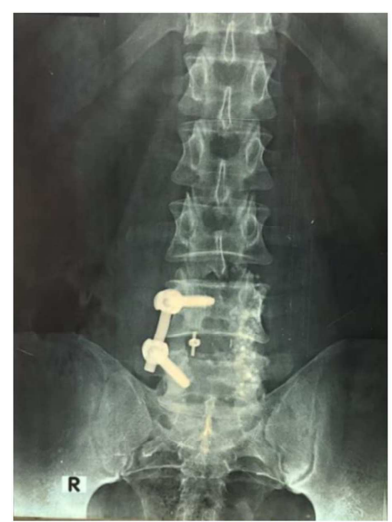

a

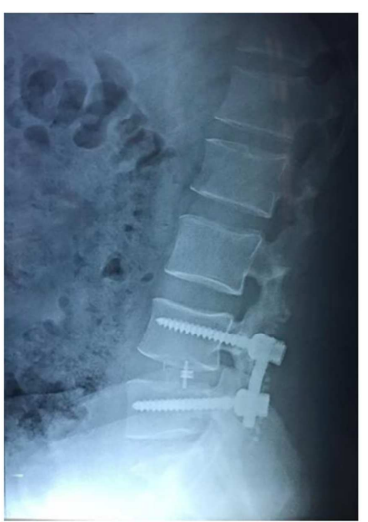

$\mathrm{b}$

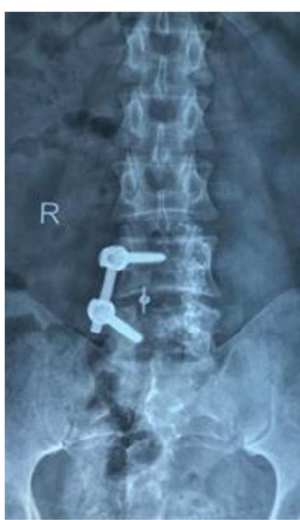

c

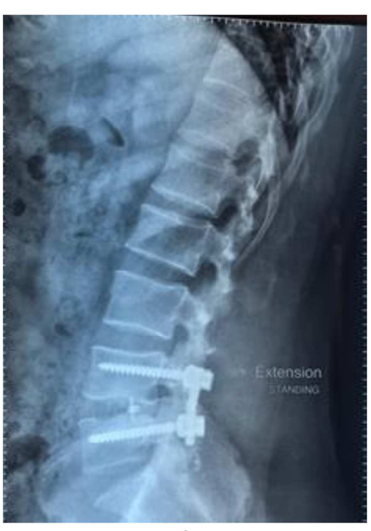

d

Figure 5. (a, b) Postoperative $x$-ray and 6 months follow up (c, d) for the 46 years old, male patient, recurrent L4-5 disc prolapse, presented with LBP and Right sciatica of 6 months'duration without improvement by conservative methods. 


\section{Discussion}

Bilateral transpedicular screws and interbody lumbar fusion has been used for treatment of degenerative lumbar spine disease, we are trying in this study to evaluate the outcome of using unilateral transpedicular screws with lumbar interbody fusion. In our study the mean VAS of the leg preoperatively was $6.79 \pm 1.30$, while the mean VAS of the back preoperatively was $6.85 \pm 1.40$. In the study of Chen DJ et al. (2018) they reported a mean preoperative VAS back of $3.31 \pm 0.81$ and the mean preoperative leg visual analogue score (VAS) of the studied patients in was found to be $5.48 \pm 1.35$ which when compared to our study was less.[4] While in the study of Kai et al. (2014) the VAS of back pain was $7.8 \pm 0.8$ and the mean preoperative leg visual analogue score (VAS) was found to be $7.4 \pm 2.8$ which goes with our patients scores.[5] In the study of Shen et al. (2016), they reported a mean preoperative VAS of $6.8 \pm 1.6$, while in the study of Işik et al. (2017) they reported a mean preoperative VAS of 8.57. [6,7]

In the study of Dahdaleh et al. (2013), they reported a mean preoperative VAS back of $5.7 \pm 2.6$, while the mean preoperative leg visual analogue score (VAS) of the studied patients in was found to be $5.7 \pm 3.1$; which were less than our patients scores. [8]

Chen et al. (2018) found in their study that the mean preoperative ODI score was $39.0 \pm 13.35$, while Kai et al. (2014) found in their study that the mean preoperative ODI score was $42.4 \pm 16 \%$. These scores were less than those recorded in our study. On the contrary Işik et al. (2017) found in their study that the mean preoperative ODI score was $63.52 \%$ which goes with the scores recorded by us. [4-6]

In our study, operation time ranged from 100-140 min (mean $118.91 \pm 12.75$ ) and by comparing these results with other studies, we realized that it was longer than the results recorded by Chen et al. (2018) $89.8 \pm 19.7$ minutes and it was close to the results recorded by Işik et al. (2017), Yang et al. (2014) and Villavicencio et al. (2015). [9, 10] On the contrary Kai et al. (2014) reported longer operative time. [5]

Regarding the blood loss intraoperative, it was ranging from 200-600 cc (mean 405.88 118.55 ). These results go with the results recorded by Kai et al. (2014), Villavicencio et al. (2014) and Aoki et al. (2012). While Chen et al. (2018), Işik et al. (2017), Yang et al. (2014) and Xue et al. (2012) recorded much less blood loss. [4-6, 9-12]

In this study the hospital stay of the patients ranged from 3-5 days. Chen et al. (2018), Kai et al. (2014) and Yang et al. (2014) reported much longer hospital stay. While Villavicencio et al. (2014) reported shorter hospital stay (0-3). On the other hand, Işik et al. (2017) reported hospital stay for their patients which was close to the results recorded in our study. $[4-6,9,10]$

Although in this study we were reporting only unilateral fixation, but when reviewing other studies reporting bilateral transpedicular screws fixation we found that unilateral fixation showed shorter operative time, less blood loss and shorter hospital stay. [4-6, 11, 12]
When comparing VAS back pain preoperatively and at postoperative intervals of 1,6 and 12 months, it showed highly significant improvement (P-value 0.000). Also when comparing VAS of leg pain preoperatively and at postoperative intervals of 1,6 and 12 months duration, it showed highly significant improvement (P-value 0.000$)$. ODI preoperatively and at postoperative intervals of 1,6 and 12 months showed also highly significant improvement (P-value 0.000). Similar data were reported in different studies showing marked improvement in the VAS of back and leg preoperatively and postoperatively. Also regarding the marked improvement in ODI score preoperatively and post operatively, it was reported in different studies. [4-6, 9, 10]

Regarding the fusion rate, at 6 months postoperative 21 of the patients $(61.8 \%)$ had fusion grade 1.9 of the patients (26.5\%) had fusion grade 2 and 4 of the patients (11.8\%) had fusion grade 3 . While at 12 months postoperative the percent of grade 1 fusion increased to become $85.3 \%$ (29 patients) and the rest were grade 2 fusions $(14.7 \%)$ with no patients with grade 3 fusion. The results reported in our study were near to those reported by Işik et al., Villavicencio et al., Xue et al. and Dahdaleh et al. [6, 8, 9, 12]

As for complications there were 2 patients $(5.9 \%)$ with incidental durotomy, 1 patient with cage migration $(2.9 \%)$ and 1 patient $(2.9 \%)$ with superficial wound infection. These results were near those reported by Yang X et al. and Aoki et al. $[10,11]$

As we can see from the previous results and the various data reported by other studies the use of unilateral pedicular screws had proven its efficacy with shorter operative time and hospital stay together with minimal blood loss. This was associated with marked improvement in clinical picture and very good fusion rate post operatively with no remarkable complication rate.

\section{Conclusion}

Unilateral pedicular screws and interbody fusion is a good modality of treatment for unilateral degenerative spine pathology, with shorter operative time and hospital stay together with minimal blood loss. This was associated with marked improvement in clinical picture and very good fusion rate post operatively with no remarkable complication rate.

\section{References}

[1] Huan Liu, Ying Xu, Si-Dong Yang, Tao Wang, Hui Wang, Feng-Yu Liu, Wen-Yuan Ding: Unilateral versus bilateral pedicle screw fixation with posterior lumbar interbody fusion for lumbar degenerative diseases A meta-analysis. Medicine, 96: 21 (e6882), 2017.

[2] Si-Dong Yang, Qian Chen, Wen-Yuan Ding, Jian-Qiang Zhao, Ying-Ze Zhang, Yong Shen, Da-Long Yang: Unilateral Pedicle Screw Fixation with Bone Graft vs. Bilateral Pedicle Screw Fixation with Bone Graft or Cage: A Comparative Study, Med Sci Monit; 22: 890-897, 2016. 
[3] Liu Z, Fei Q, Wang B, Lv P, Chi C, Yang Y, Zhao F, Lin J, Ma Z.: A meta-analysis of unilateral versus bilateral pedicle screw fixation in minimally invasive lumbar interbody fusion. PLoS One. 6; 9 (11): e111979, 2014.

[4] Chen DJ, Yao C, Song Q, Tang B, Liu X, Zhang B, Dai M, Nie T, Wan Z.: Unilateral versus Bilateral Pedicle Screw Fixation Combined with Transforaminal Lumbar Interbody Fusion for the Treatment of Low Lumbar Degenerative Disc Diseases: Analysis of Clinical and Radiographic Results. World Neurosurg; 115: e516-e522, 2018.

[5] Kai Z, Wei S, Chang-qing Z, Hua L, Wei D, You-zhuan X, Xiao-jiang S, Jie Z.: Unilateral versus bilateral instrumented transforaminal lumbar interbody fusion in two-level degenerative lumbar disorders: a prospective randomised study. International orthopaedics.; 38 (1): 111-6, 2014.

[6] Işik HS, Okutan Ö, Yildirim T, Akpinar E, Yilmaz A.: Comparison of Unilateral versus Bilateral Pedicle Screw Fixation in Transforaminal Lumbar Interbody Fusion for Single Level Lumbar Degenerative Diseases and Review of Literature. Turk Neurosurg, vol. 28, no. 5, 731-739, 2018.

[7] Shen X, Wang L, Zhang H, Gu X, Gu G, He S.: Radiographic Analysis of One-level Minimally Invasive Transforaminal Lumbar Interbody Fusion (MI-TLIF) With Unilateral Pedicle Screw Fixation for Lumbar Degenerative Diseases. Clin Spine Surg.; 29 (1): E1-8, 2016.
[8] Dahdaleh NS, Nixon AT, Lawton CD, Wong AP, Smith ZA, Fessler RG.: Outcome following unilateral versus bilateral instrumentation in patients undergoing minimally invasive transforaminal lumbar interbody fusion: a single-center randomized prospective study. Neurosurg Focus. Aug; 35 (2): E13, 2013.

[9] Villavicencio AT, Serxner BJ, Mason A, Nelson EL, Rajpal S, Faes N, Burneikiene S.: Unilateral and bilateral pedicle screw fixation in transforaminal lumbar interbody fusion: radiographic and clinical analysis. World Neurosurg; 83 (4): 553-9, 2015.

[10] Yang X, Wang H, Zhao Q, Xu H, Liu P, Jin Y.: A comparison of unilateral and bilateral pedicle screw fixation combined with transforaminal lumbar interbody fusion for lumbar degenerative diseases. Chin Med J (Engl); 127 (20): 3592-6, 2014.

[11] Aoki Y, Yamagata M, Ikeda Y, Nakajima F, Ohtori S, Nakagawa K, Nakajima A, Toyone T, Orita S, Takahashi K.: A prospective randomized controlled study comparing transforaminal lumbar interbody fusion techniques for degenerative spondylolisthesis: unilateral pedicle screw and 1 cage versus bilateral pedicle screws and 2 cages. J Neurosurg Spine; 17 (2): 153-9, 2012.

[12] Xue H, Tu Y, Cai M.: Comparison of unilateral versus bilateral instrumented transforaminal lumbar interbody fusion in degenerative lumbar diseases. Spine J.; 12 (3): 209-15, 2012. 